\title{
Recomendaciones de higiene postural para Sonografistas Cardio Vasculares No Médicos
}

Aguilar Q, Elibeth A. ${ }^{1}$

Restrepo L, Patricia M. ${ }^{2}$

Sánchez B, Ennio B.

\section{Correspondencia}

Elibeth Aguilar Quiroz

elibethaguilar@gmail.com

'Licenciada en Tecnología y Terapia Cardiopulmonar, Sonografista Cardiovascular No Médico.

2Licenciada en Enfermería, Sonografista Cardiovascular No Médico.

${ }^{3}$ Licenciado en Fisioterapia, Especialista en fisioterapia cardiovascular, Hospital Universitario de Caracas.

Recibido: 10/10/2021

Aceptado: 13/10/2021

En línea: 15/12/2021

Citar como: Aguilar Q Elibeth, Restrepo L Patricia, Sanchez B Ennio: Recomendaciones de higiene postural para sonografistas no médicos (SCVNM). RETIC. 2021 (Diciembre); 4 (3): 50-52. doi: 10.37615/retic.v4n3a14

Cite this as: Aguilar Q Elibeth, Restrepo L Patricia, Sanchez B Ennio: Postural work recommendations for non medical sonographers. RETIC. 2021 (Diciembre); 4 (3): 5052. doi: $10.37615 /$ retic.v4n3a14

\section{Palabras clave}

$\triangleright$ Ultrasonido.

$\triangleright$ Higiene postural.

$\triangleright$ Prevención

\section{Keywords}

\section{Cuerpo del artículo}

Los trastornos musculoesqueléticos relacionados con el trabajo (TMERT) son lesiones dolorosas que afectan los músculos, nervios y tendones de hasta el 90\% de los sonografistas $1.20 \%$ de los sonografistas sintomáticos sufren lesiones que pusieron fin a su carrera. Los síntomas de TMRT tienen una incidencia de aparición de un 15\% a los seis meses del inicio del empleo, aumentando a $45 \%$ después de tres años y hasta $72 \%$ después de diez años1, con un predominio sintomático en la región cervical, lumbar, hombros y manos (figura 1).

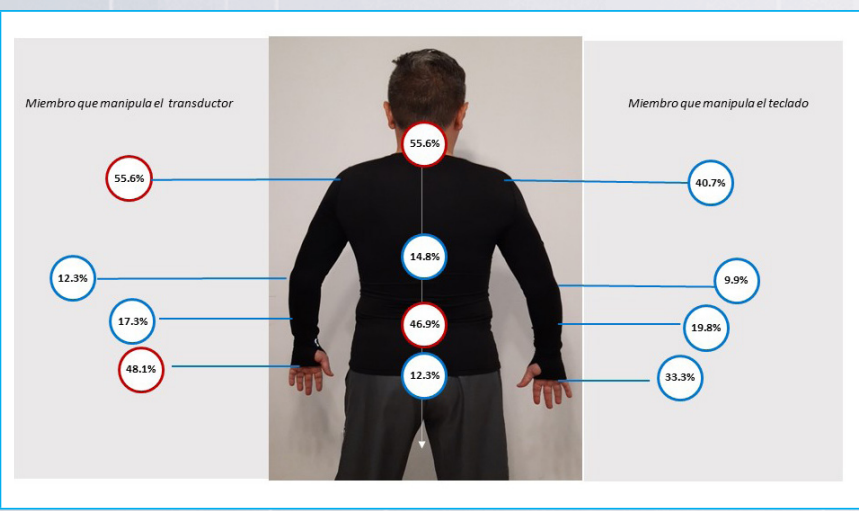

Figura 1. Áreas más frecuentes de dolor de los sonografistas.
Algunos de los factores que pueden predisponer la aparición de lesiones musculoesqueléticas en sonografistas (tabla 1) son las posiciones estáticas o incómodas y los movimientos que se realizan al utilizar el transductor o durante la ubicación de pacientes y equipo ${ }^{2}$, así como la presión constante y continua por largos períodos de tiempo durante la realización de las pruebas ${ }^{3}$ (tabla 2). A continuación, se presentarán algunos principios de higiene postural que debe tener el sonografista cardiovascular no médico SCVNM para evitar los TMRT más frecuentes:

\begin{tabular}{|l|}
\multicolumn{1}{|c|}{ TMERT más comunes entre ecografistas } \\
\hline - Síndrome del túnel carpiano \\
- Epicondilitis \\
- Tendinitis del manguito rotador \\
- Lumbalgia \\
- Síndrome cervical por tensión \\
\hline
\end{tabular}

Tabla 1. TMERT más comunes entre ecografistas.

Mejores prácticas de higiene postural al momento de realizar la exploración con ultrasonido 4.5 .

- A nivel del hombro, las posturas que deben evitarse son aquellas estáticas que combinan abducción mayor de $45^{\circ}$ con la rotación interna. Para ello, es aconsejable que el sonografista esté muy cercano al paciente, y de ser posible, descansar el codo en la camilla del paciente (si el SCVNM está sentado) (figura 2).

- La posición del codo del sonografista debe estar cercana a los $90^{\circ}$ de flexión.

- La mano que sujeta el transductor debe estar alineada con el antebrazo, y evitar la flexión o la extensión exagerada, sobre todo cuando se usa la 
- Trukipedia

Truco 01

\begin{tabular}{|l|c|c|}
\hline \multirow{2}{*}{\multicolumn{1}{c}{ Posiciones y tareas más agravantes }} & \% de posiciones y tareas que fueron consideradas agravantes o muy agravantes \\
\cline { 2 - 3 } & Agravantes & Muy agravantes \\
\hline Aplicar presión con el transductor & 22,6 & 60,8 \\
\hline Abducción sostenida con el hombro & 24,9 & 58,4 \\
\hline Rotación sostenido de cuello o tronco & 25 & 47,8 \\
\hline Rotación repetitiva de cuello o tronco & 25,4 & 42,4 \\
\hline Levantar o asistir pacientes & 20,8 & 19,2 \\
\hline Transportar equipos & 15,2 & 14,6 \\
\hline Posición bípeda del sonografista & 17,5 & 10,5 \\
\hline Posición sedente del sonografista & 11,2 & 5,1 \\
\hline Realización de las mediciones & 5,4 & 2,7 \\
\hline
\end{tabular}

Tabla 2. Posiciones y tareas más agravantes de los síntomas reportados por los sonografistas.

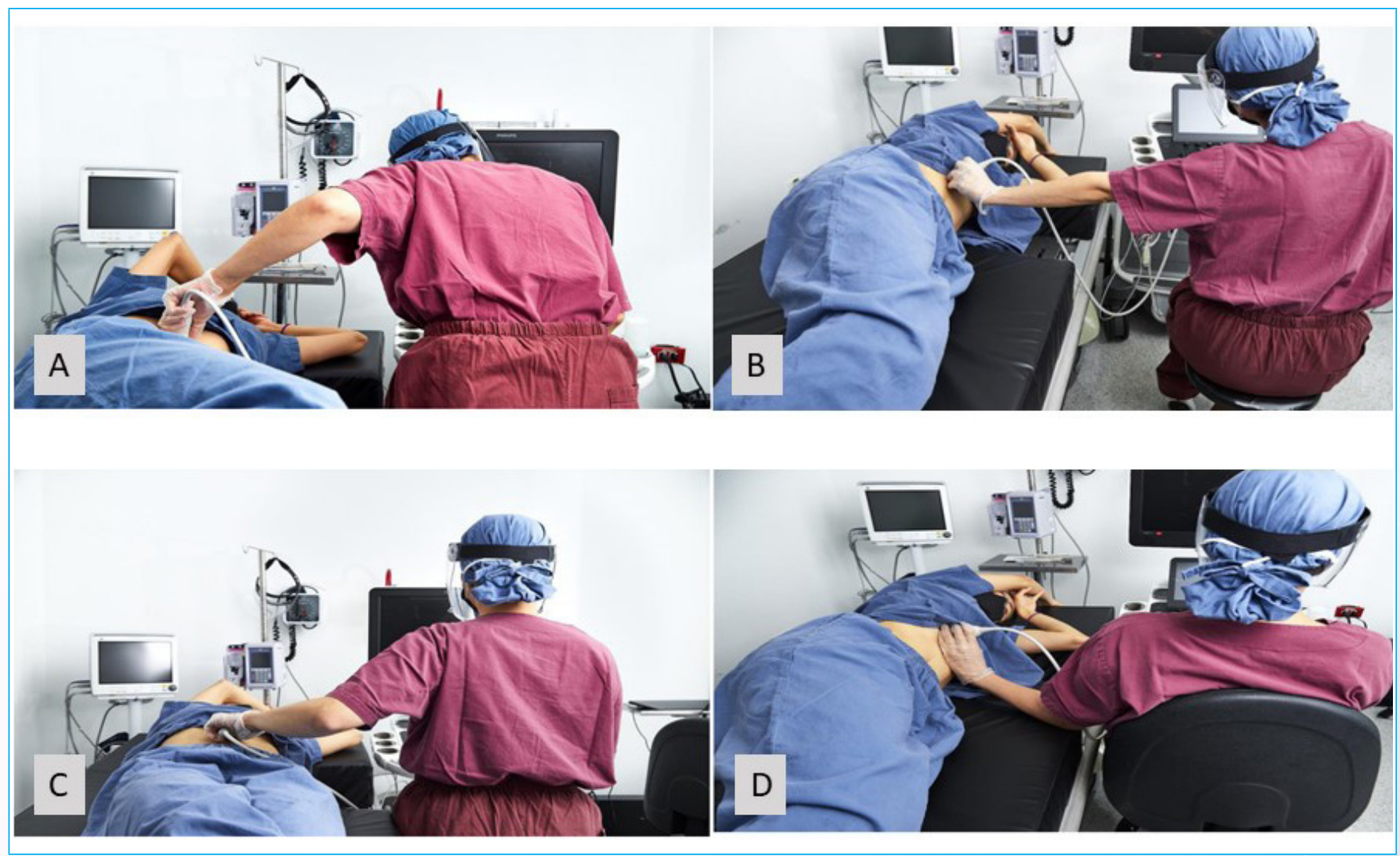

Figura 2. Posturas adecuadas e inadecuadas en posición bípeda y sedente.

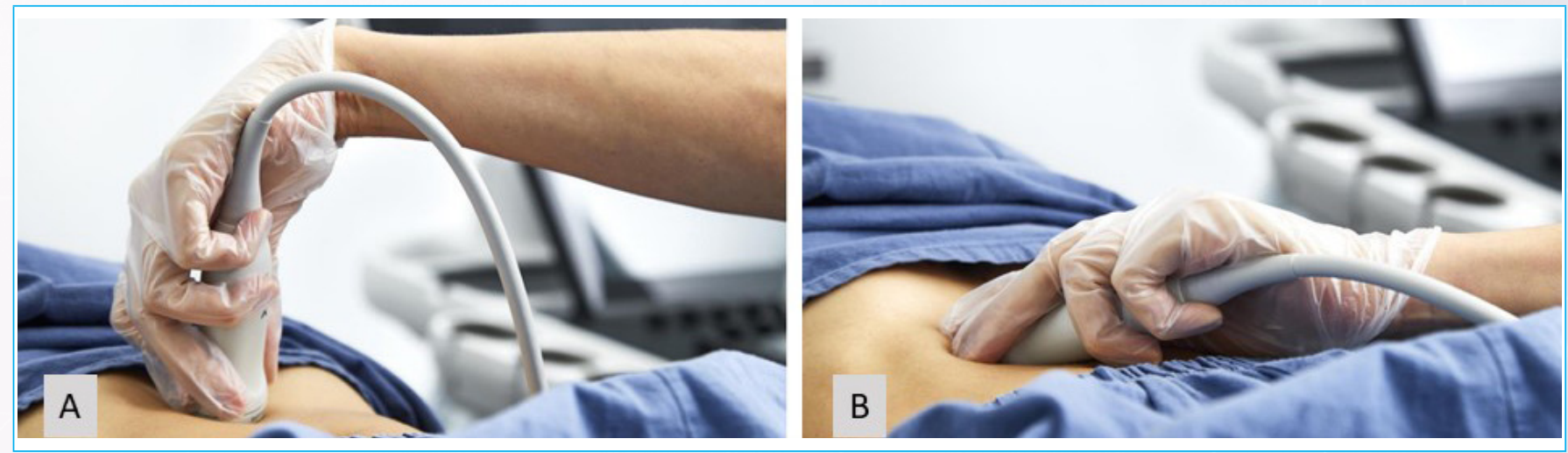

Figura 3. Posturas adecuadas e inadecuadas de muñeca. 
fuerza al momento de la exploración. Lo correcto o ideal es que el agarre del transductor sea completo de manera que la mano pueda extenderse por el borde ancho e inferior del transductor. Se debe apoyar los dedos sobre la piel del paciente para lograr una mayor estabilidad y a la vez limitar la presión (figura 3).

- Se debe evitar posiciones estáticas continuas de rotación y flexión del cueIlo. Para ello, lo mejor es colocar el equipo de ecocardiografía lo más cerca del paciente.

- En la posición sedente, el SCVNM deberá apoyar el antebrazo o el codo de la mano no exploratoria sobre el apoyabrazos o sobre el equipo, a fin de disminuir la tensión acumulada sobre cuello y hombro. Al momento de utilizar el "TrackBall", la postura de la muñeca debe permitir la alineación de la mano y del antebrazo.

- Las pausas activas implican detener la actividad laboral, para favorecer la recuperación muscular, a fin de disminuir la incidencia de aparición de molestias o afecciones musculoesqueléticas durante la ejecución del trabajo.

\section{Ideas para recordar}

- Los TMRT pueden acortar los años libres de discapacidad de un SCVNM.

- Los cuidados posturales pueden prevenir los TMRT.

\section{Bibliografía}

1. SDMS: Society of Diagnostic Medical Sonography [Internet]. EE. UU: SDMS; 2019 [citado 3 sep. 2021]. WRMSD y ecografía. Disponible en: https://www. sdms.org/resources/careers/work-related-musculoskeletal-disorders.

2. NIOSH: Instituto Nacional para la Seguridady Salud Ocupacional [Internet]. EE. UU: NIOSH; 2006 [citado 3 sep. 2021]. Prevención de trastornos musculoesqueléticos en la realización de ecografías. Disponible en: https://www.cdc. gov/spanish/niosh/

3. Society of Diagnostic Medical Sonography. Industry Standards for the Prevention of Work-Related Musculoskeletal Disorders in Sonography. Journal of Diagnostic Medical Sonography [Internet] 2017, 33(5):371-391[citado 7 Sep. 2021]. Disponible en: https://journals.sagepub.com/doi/ full/10.1177/8756479317725531

4. Joan PB, Carolyn T. The Importance of an Ergonomic Workstation to Practicing Sonographers. J Ultrasound Med [Internet].2013 [Citado 8 Sep. 2021];32(8):1363-75. doi: 10.7863/ultra.32.8.1363.

5. Evans K, Roll S, Baker J. Work-Related Musculoskeletal Disorders (WRMSD) Among Registered Diagnostic Medical Sonographers and Vascular Technologists: A Representative Sample. J Diagn Medical Sonography 2009; 25:298-99. Disponible en: https://journals.sagepub.com/ doi/10.1177/8756479309351748 